\title{
The Clinical Significance and Potential Therapeutic Role of GPx3 in Tumor Recurrence after Liver Transplantation
}

\author{
Xiang Qi ${ }^{1}$, Kevin Tak-Pan Ng${ }^{1}$, Yan Shao ${ }^{1}$, Chang Xian Li ${ }^{1}$, Wei Geng ${ }^{1}$, Chang Chun Ling ${ }^{1}$, Yuen Yuen Ma1, \\ Xiao Bing Liu1, Hui Liu¹, Jiang Liu1, Wai Ho Yeung1, Chung Mau Lo1,2, Kwan Man ${ }^{1,2} \bowtie$ \\ 1. Department of Surgery, The University of Hong Kong, Pokfulam, Hong Kong. \\ 2. Collaborative Innovation Center for Diagnosis and Treatment of Infectious Diseases, China. \\ $\square$ Corresponding author: Prof. Kwan Man; Address: L9-55, Lab Block, 21 Sassoon Road, Pokfulam, Hong Kong; Email: kwanman@hku.hk; Telephone: \\ 852-39179646; Fax: 852-39179634.
}

(1) Ivyspring International Publisher. Reproduction is permitted for personal, noncommercial use, provided that the article is in whole, unmodified, and properly cited. See http://ivyspring.com/terms for terms and conditions.

Received: 2016.05.01; Accepted: 2016.06.08; Published: 2016.08.08

\begin{abstract}
Background and Aims: Our previous study showed that small-for-size liver graft may provide favorable micro-environment for tumor growth. GPx3, an anti-oxidant, not only attenuates oxidative stress, but also suppresses liver tumor growth in our recent study. Here, we aimed to characterize the clinical significance and explore the functional role of GPx3 in HCC recurrence after liver transplantation.

Methods: To explore the association between GPx3 expression and HCC invasiveness, a rat orthotopic liver transplantation model with tumor development was established. To investigate the clinical relevance of GPx3, $105 \mathrm{HCC}$ patients who underwent liver transplantation were recruited. The suppressive role of GPx3 in HCC cells was studied using wound healing, Matrigel invasion assay and lung metastasis model. The real-time intravital imaging system was applied to directly visualize the tumor cells invasion in a living animal. The underlying mechanism was further explored.

Results: GPx3 was identified as a down-regulated protein in small-for-size liver graft and significantly associated with invasive phenotype of tumor growth in a rat model. Plasma GPx3 was significantly lower in small-for-size graft group post-transplantation (dayl: 33 vs 1147; day3: 3209 vs 4459; day7: 303 vs 2506; $\mathrm{mU} / \mathrm{mL}, P<0.05$ ) in rat model. Clinically, the plasma GPx3 was significantly lower in the recipients with $\mathrm{HCC}$ recurrence post-transplantation (dayl: $4.16 \mathrm{vs} 8.99 \mu \mathrm{g} / \mathrm{mL}, P<0.001$; day7: $3.86 \mathrm{vs} 9.99 \mu \mathrm{g} / \mathrm{mL}$, $P<0.001)$. Furthermore, lower plasma GPx3 was identified as an independent predictor $(H R=4.528$, $P=0.046$ ) for poor overall survival post-transplantation. Over-expression of GPx3 significantly suppressed migration, invasiveness and metastasis of HCC cells. Real-time intravital imaging showed that GPx3 significantly suppressed HCC invasiveness in a live animal. GPx3 suppressed the tumor invasiveness through inhibition of JNK-cJun-MMP2 pathway.

Conclusion: GPX3 may possess prognostic and therapeutic value for HCC patients after liver transplantation.
\end{abstract}

Key words: GPx3; HCC invasiveness; intravital imaging; liver transplantation.

\section{Introduction}

The higher incidence of tumor recurrence after living donor liver transplantation (LDLT) compared with deceased donor liver transplantation (DDLT) was reported in hepatocellular carcinoma (HCC) patients [1-4]. The liver grafts from living donor are always small-for-size for adult recipients. From our previous publication $[5,6]$, both of our clinical and animal studies showed that small-for-size liver graft provides favorable environment for tumor recurrence after transplantation. Recently, we also demonstrated that GPx3, an anti-oxidant, not only attenuates oxidative stress, but also suppresses liver tumor 
growth [7]. In our preliminary study, we found that GPx3 was significantly down-regulated in small-for-size liver graft, in which more invasive tumor cells were observed. Thus, down-regulation of GPx3 may be one of the reasons for more aggressive tumor behavior in small-for-size liver graft. Based on those findings, we hypothesize that GPx3 may possess prognostic and therapeutic value in tumor recurrence after liver transplantation, especially for transplantation using a small-for-size liver graft. Therefore, Investigation of the clinical significance of GPx3 on tumor recurrence after transplantation, and exploration of the suppressive role of GPx3 in HCC invasiveness, may help for developing novel therapeutic strategy targeting at HCC recurrence after liver transplantation.

Glutathione peroxidase 3 (GPx3) is a selenium-dependent enzyme which functions in detoxification of reactive oxygen species (ROS) to protect the organ from overwhelming oxidative stress [8]. It catalyzes the reduction of hydrogen peroxidase, organic peroxidase and lipid peroxidase by reduced glutathione. According to the reports, GPx3 are always down-regulated in several types of cancer due to genomic deletion or epigenetic alteration, such as prostate cancer [9], gastric cancer [10] and Barrett's adenocarcinoma [11]. Moreover, the circulating GPx3 was identified to be significantly lower in the patients with glioblastoma compared with non-tumor patients [12]. Furthermore, our previous study has shown that GPx3 suppressed the proliferation of HCC cells [7]. The role of GPx3 in HCC invasiveness needs further exploration.

With the development of imaging technology, the modality of molecular intravital imaging currently enables us to directly see the tumor cells in a live animal. Optical technique of functional imaging has well developed in clinical situation, including magnetic resonance imaging (MRI), positron emission tomography (PET) or computed tomography (CT). However, the major challenge in such technique is the limited penetration depth imposed by tissue turbidity, which avoids the possibility of utilizing high resolution microscope [13]. Here, we utilized a novel in vivo imaging system using dorsal window chamber which could overcome such limitation. The intravital imaging of dorsal window chamber has been already applied in detection of vascularization with the advantages of dynamically observation and high resolution in animal model [14]. The establishment of new platform of imaging would allow us to observe the tumor behavior with high resolution in a live animal.

In the current study, we aimed to explore the prognostic role and therapeutic value of GPx3 on tumor recurrence after liver transplantation for HCC patients. We firstly studied the association between GPx3 expression and HCC invasiveness in a rat orthotopic liver transplantation model. Then we investigated the clinical significance of GPx3 in HCC patients after liver transplantation. After that, we examined the functional role of GPx3 in HCC invasiveness and further explored the underlying mechanism. We hoped that our study could provide evidences to explore novel therapeutic strategy targeting at HCC recurrence after liver transplantation.

\section{Material and Methods}

\section{Study design}

The study consisted of three parts. In the first part, the association between GPx3 expression and aggressiveness of liver cancer was investigated in a rat orthotopic liver transplantation model and further validated in clinical samples (Figure S1A). In the second part, the tumor suppressive effect of GPx3 on tumor invasiveness and metastasis will be studied in vitro and in vivo (Figure S1B). In the third part, the underlying mechanisms of anti-invasiveness effect of GPx3 will be further explored in HCC cells and validated in clinical samples and animal model (Figure S1C).

\section{Clinical specimens}

The study was approved by the Institutional Review Board of the University of Hong Kong / Hospital Authority Hong Kong West Cluster. One hundred and five HCC patients who had undergone liver transplantation between December 2000 and May 2010 were recruited from Department of Surgery, Queen Marry Hospital, The University of Hong Kong. The plasma samples at different time points were collected from recipients and stored at $-80^{\circ} \mathrm{C}$. The plasma level of human GPx3 was detected using ELISA Kit (AdipoGen Inc, Korea) according to instruction manual. The median age of the recipients was 54 (30-67) years old. Meanwhile, the median age of the donors was 38 (18-71) years old. The median of follow up time is 5 years ( 3 months - 10 years). Ninety-two recipients (87\%) were male and 64 donors $(61 \%)$ were female. Seventy-two donor grafts (68\%) were small-for-size (the graft radio $\leq 60 \%$ ). Seventy-nine liver grafts $(75 \%)$ were from living donors. The study was approved by the Ethics Committee of the University of Hong Kong.

\section{Rat Liver Transplantation models}

Male inbred Buffalo rats (body weight: 300-350g) were used as donors and recipients for transplantation. All the rats were kept with access to 
water and chow in a standard animal laboratory. They were housed with a 12-hour light/dark cycle and fasted 12 hours before transplantation. All operations were performed under clean conditions. The study had been licensed according to Animal (Control of Experiments) Ordinance Chapter 340 by the Department of Health, Hong Kong Special Administrative Region. (ref.: (11-632) in DH/HA\&P/8/2/3 Pt. 31).

A rat non-arterialized orthotopic liver transplantation model was established using whole graft and small-for-size graft as donors [4, 5]. Briefly, the recipients were injected with a rat hepatoma cell line (McA-RH7777, $2 \times 10^{5} / 200 \mu \mathrm{L}$ ) via the portal vein after reperfusion to mimic the clinical situation of remained tumor cells in circulation homing to the liver graft. The median graft ratio in small-for-size graft group (the ratio of graft weight to recipient liver weight) was $55 \%(48-60 \%)$. The rats were sacrificed at days 1, 3, 7, 14 and 21 after transplantation for collecting liver tissues and blood samples. Liver tumor tissues and adjacent non-tumor tissues were collected at days 14 and 21 post-transplantation.

\section{Identification of proteomic expression profile by 2-dimensional electrophoresis (2-DE) and Mass spectrometry (MS)}

The whole blood samples were obtained from caudal vein of rats and collected into vacutainer containing EDTA. After centrifuged at $3000 \mathrm{rpm}$ for $10 \mathrm{~min}$, the plasma could be separated and stored at $-80^{\circ} \mathrm{C}$ for further investigation. In first dimension electrophoresis, $400 \mu \mathrm{g}$ plasma samples were loaded onto linear immortalized $\mathrm{pH}$ gradient (IPG) strips ( $\mathrm{pH}$ 3-10 L, $240 \times 3 \times 0.5 \mathrm{~mm} 3$, BioRad) without any pretreatment. The second dimension was performed on $12 \%$ SDS-PAGE in a protean-plus DodecaTM Cell (BioRad). After visualized by silver-staining technique, the differential protein spots were excised using biopsy punches and transferred to $1.5 \mathrm{~mL}$ Eppendorf tube for Mass-spectrometry (MS) analysis using Ultraflex 2 TOF/TOF mass spectrometer (Bruker Daltonics).

\section{Real time quantitative reverse transcription polymerize chain reaction ( $q R T-P C R)$ and Western-blot}

The expression level of GPx3 was detected by qRT-PCR and Western-blot as previously described $[7,15,16]$.

\section{Immunohistochemistry (IHC) staining}

Sections of paraffin-embedded tissue $(4 \mu \mathrm{m})$ were stained as previously described [17]. For quantification, the sections of each tumor were evaluated independently by two different investigators. The intensity of immunostaining was scored as: weak (1), moderate (2), strong (3) and extremely strong (4). An immunohistochemistry score (ranging from 0 to 4 ) was calculated as follows: (staining intensity $\times$ percentage of positively labeled cells) / 100.

\section{Enzyme-linked immunosorbent assay (ELISA) and GPx3 enzyme activity assay}

The plasma samples of clinical patients were tested by ELISA [18] and the rat plasma level of GPx3 was detected by enzyme activity assay as previously described [7].

\section{Construction of stable HCC cell line with over-expression of GPx3}

MHCC97L was transduced with full-length human GPx3 gene as previously described [7].

\section{Wound healing assay and Matrigel invasion assay}

To investigate the migration of HCC cells, wound healing assay was performed as previously described [19]. To examine the invasiveness of HCC cells, Matrigel invasion assay was performed as previously described $[4,19]$.

\section{Nude mice orthotopic liver cancer model}

We implanted the tumor tissue cubes into the left liver lobes of nude mice as described previously [20].

\section{Real-time intravital imaging using dorsal window chamber}

The dorsal window chamber (DWC) consists of two titanium frames that marry together to form a saddle on the back of the nude mouse and is attached using spacers, bolts and fastening nuts. A 'chamber' is formed after a transparent glass cover slip placed onto the attached saddle covering the exposed fascia containing vessels and secured using a sterile removable ' $C$ ' clip. The nude mice ectopic liver cancer model was established using liver cancer cells (MHCC97L). MHCC97L cells were labeled with fluorescence reporter (GFP) during transduction and then injected subcutaneously into the window chamber from the opposing side to the glass cover-slip. The tissue structure surrounding the tumor nodule, such as collagen, would be visualized through two photons secondary harmonic generation. The process of cancer cells invasiveness and metastasis would be kinetically observed through 3D-images reconstruction under CZ LSM 710 confocal system. The excitation wavelength was $800 \mathrm{~nm}$ (two-photon system). The emission wave lengths of GFP from tumor cells were $500-550 \mathrm{~nm}$. The emission 
wave lengths from connective tissue were $385-425 \mathrm{~nm}$. The objective lens was $20 \times$.

\section{Lung metastasis model with tumor cells injection via tail vein}

The nude mice were assigned into two groups. The liver cancer cell lines MHCC97L-pCDH or MHCC97L-pCDH-GPx3 $\left(2 \times 10^{6} / 100 \mu 1 \quad\right.$ PBS $)$ were injected through tail vein in each group. The mice with lung metastasis were visualized using in vivo imaging system (Perkin Elmer IVIS Spectrum). The background fluorescence noises could be distinguished from the positive signals through unmixing process. The lung metastasis will be finally confirmed by HE staining of tissue sections when the nude mice are sacrificed.

\section{Statistical analysis}

The Chi-square test was used to compare categorical data. Paired or unpaired $\mathrm{T}$ test were adopted to compare continuous variables. The median of plasma GPx3 was set as cut-off point by with all the patients were segregated into two groups: plasma GPx3 high and low group. Kaplan-Meier survival analysis was performed to compare the survival outcome of HCC patients. The significant difference between survival outcomes was detected by log-rank test. Cox proportional hazard regression model was used to identify predictors for overall survival. The variables were selected into final equation in multivariable analysis according to forward stepwise selection procedure. $\mathrm{P}<0.05$ was considered as statistically significant. Calculation was made using SPSS computer software version 16 (SPSS Inc, Chicago, IL, USA).

\section{Results}

GPx3 was identified as a down-regulated protein in small-for-size liver graft in a rat liver transplantation model with tumor development

Our previous study showed that small-for-size liver graft provides favorable micro-environment for tumor growth $[5,6]$. In the current study, we identified that GPx3 was significantly down-regulated in small-for-size liver graft in a rat orthotopic liver transplantation model (Table S1). Recently, we also demonstrated that GPx3 not only attenuates oxidative stress, but also suppresses the tumor growth in our recent study [7]. Therefore, GPx3 was selected for the further investigation in the current study. Other potential protein candidates are being tested.

Invasive tumor growth was associated with down-regulation of GPx3 in small-for-size liver graft

The down-regulation of GPx3 in small-for-size liver graft was further confirmed in another independent set of animal samples. Plasma GPx3 was significantly lower in small-for-size graft group compared with whole graft group at different time points after liver transplantation (Figure 1A). The same trend of the kinetic change of GPx3 expression was also observed in intra-graft GPx3 expression (Figure 1B). The expression of GPx3 within tumor tissues was significantly down-regulated in small-for-size graft in both mRNA (Figure 1C) and protein level (Figure 1D). Tumor cells were more invasive in small-for-size liver graft in which the GPx3 was confirmed to be down-regulated (Figure 1E and 1F). It implied that down-regulation of GPx3 might be associated with tumor invasiveness in small-for-size liver graft.

\section{Lower plasma GPx3 was correlated with more recurrence and poor survival of HCC patients after liver transplantation}

In order to explore the clinical significance of GPx3, 105 HCC patients who had undergone liver transplantation were recruited in our study. Plasma GPx3 was significantly lower in the HCC recipients with tumor recurrence after liver transplantation (Figure 2A). Moreover, plasma GPx3 was also correlated with graft size. The lower plasma GPx3 at day 1 post-transplantation was mainly observed in the patients with small-for-size liver graft (Figure 2B). The median of plasma GPx3 was selected as the cut-off point, by which all the patients were segregated into two groups: plasma GPx3 high group and low group. The patients with lower plasma GPx3 had both significantly poorer overall survival and disease free survival (Figure $2 \mathrm{C}$ and 2D). Mean estimated survival time of the patients with lower plasma GPx3 at day 1 post-transplantation was significantly shorter compared to those with higher GPx3 $(67.42 \pm 6.89$ vs $105.57 \pm 3.35$ months for overall survival; $55.21 \pm 8.26$ vs $102.88 \pm 4.08$ months for disease free survival). Mean estimated survival time of the patients with lower plasma GPx3 at day 7 post-transplantation was also significantly shorter compared with higher GPx3 group (77.26 \pm 7.45 vs $101.06 \pm 5.62$ months for overall survival; $63.64 \pm 8.55$ vs $95.63 \pm 6.99$ months for disease free survival). 


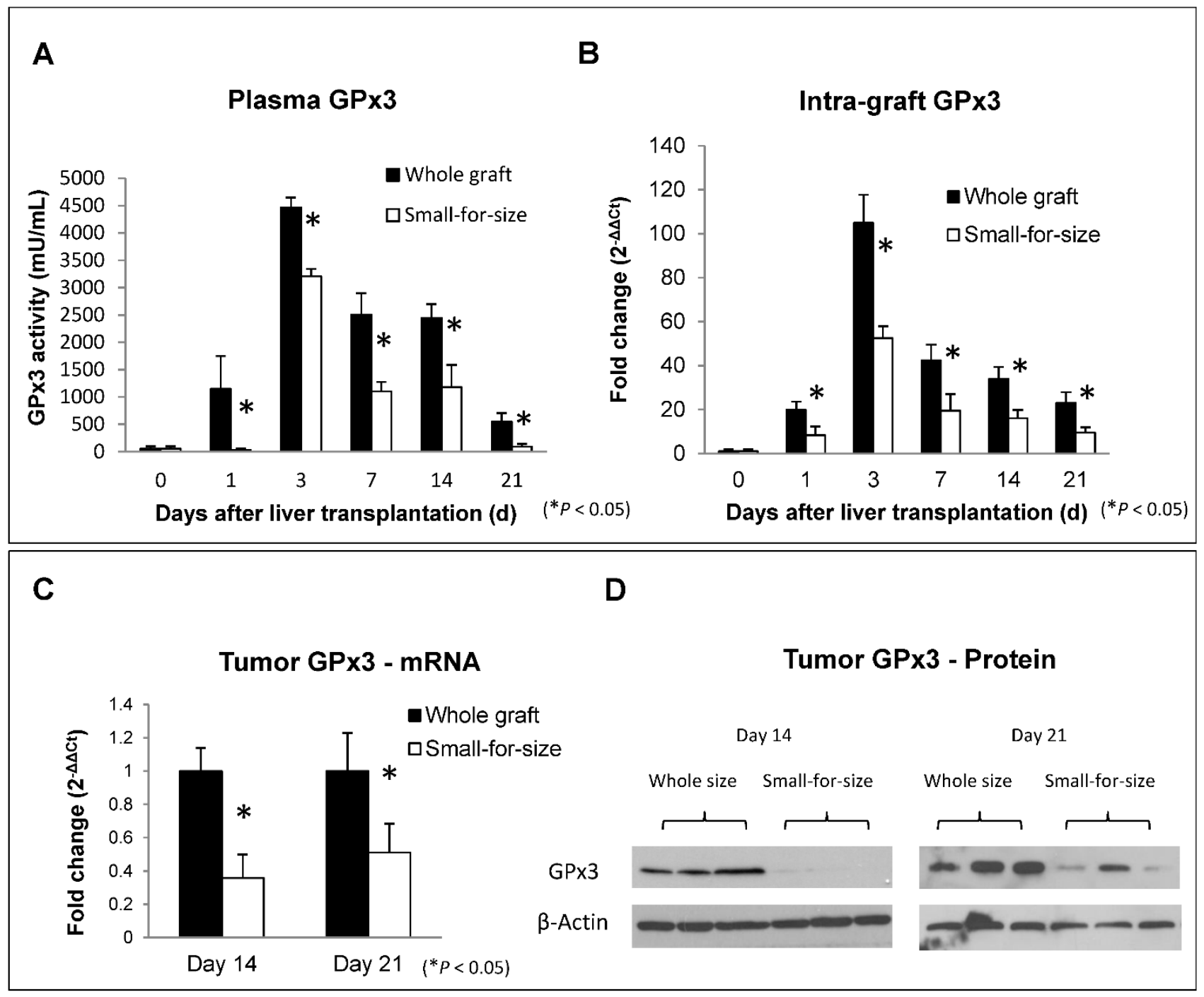

E

\section{$\mathbf{F}$}

Tumor invasion

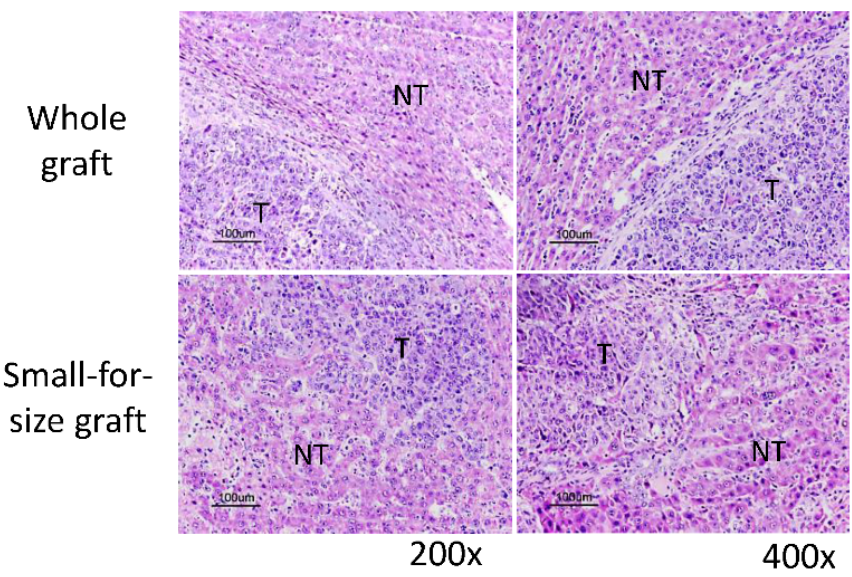

GPx3 expression

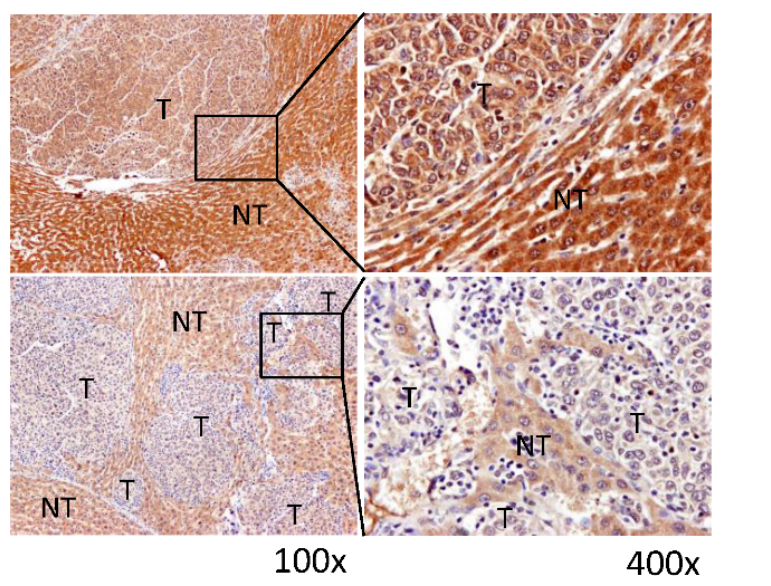

Figure 1. Expression of GPx3 was significantly down-regulated in small-for-size liver graft in which tumor cells were more invasive. (A) Plasma GPx3 was significantly lower at different time points post-transplantation in small-for-size liver graft compared with whole graft, $P<0.05$. The error bar represented as mean \pm SD. (B) Intra-graft GPx3 expression was also significantly down-regulated in small-for-size grafts compared with whole grafts, $P<0.05$. The error bar represented as mean \pm SD. Expression of GPx 3 was significantly down-regulated within tumor tissues in small-for-size graft in (C) mRNA and (D) protein level. (E) H\&E staining showed that tumor cells were more invasive in small-for-size liver graft. (F) IHC staining showed intra-hepatic spread of tumor nodules in small-for-size liver graft in which GPx3 was significantly down-regulated. 
A

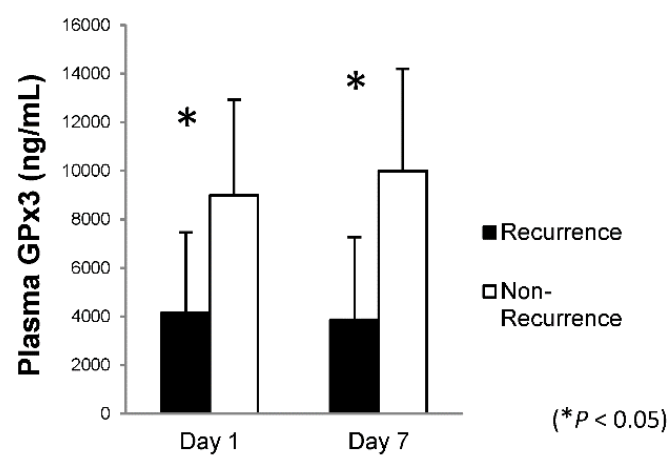

B

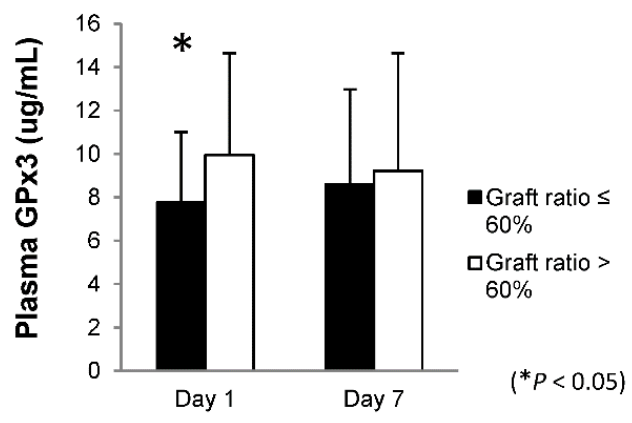

C

Overall survival

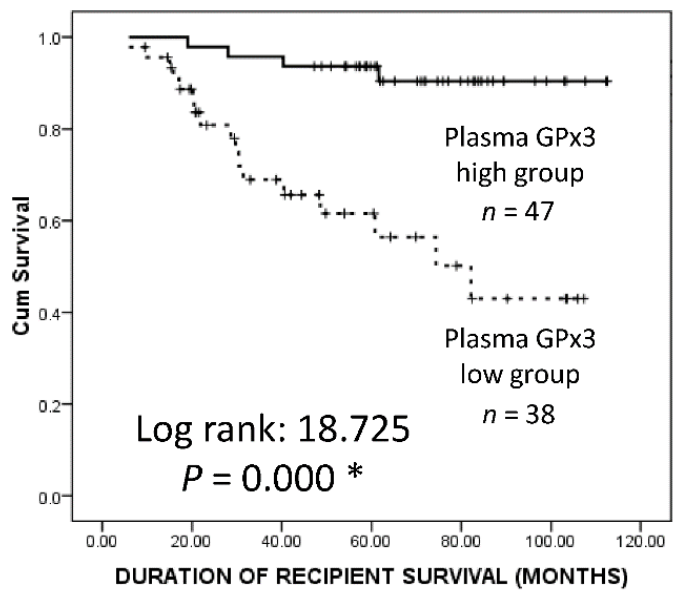

Day1

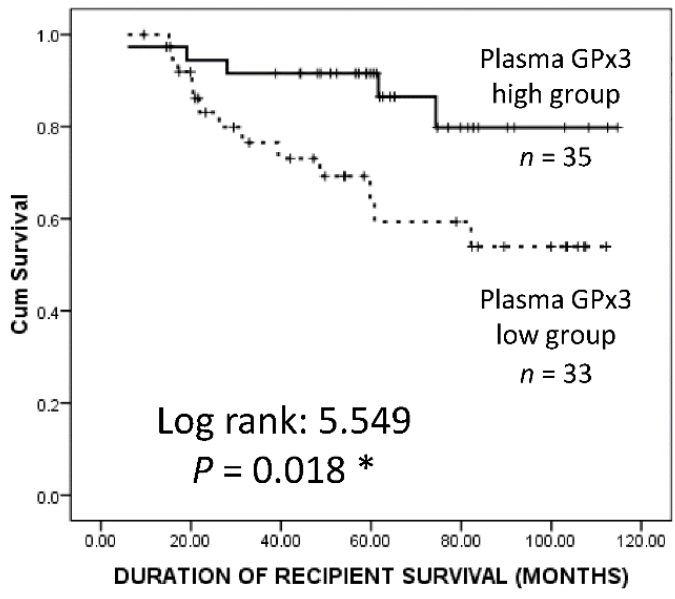

Day7

D

Disease free survival
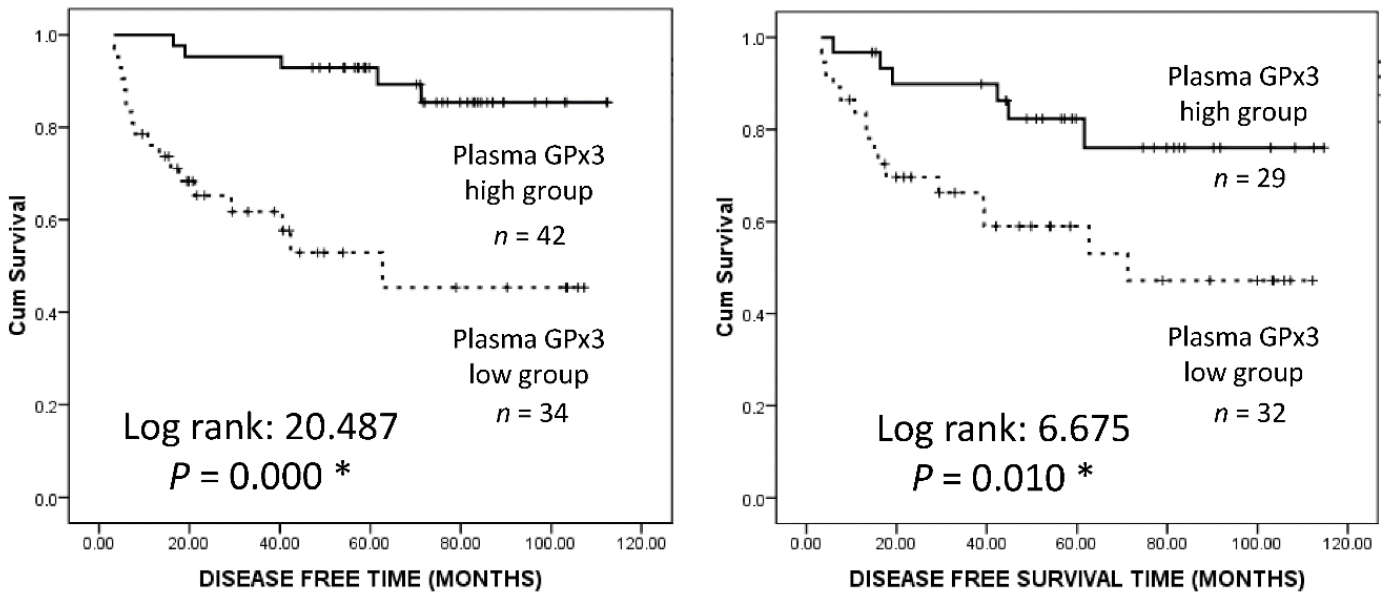

Day1

Day7

Figure 2. Lower plasma GPx3 significantly correlated with more recurrence and poor survival of HCC patients after liver transplantation. (A) Plasma GPx3 was significantly lower in the recipients with tumor recurrence after liver transplantation, $P<0.05$. The error bar represented as mean \pm SD. (B) Lower plasma GPx3 at day 1 post-transplantation significantly correlated with small graft size, $P<0.05$. The error bar represented as mean $\pm \mathrm{SD}$. Lower plasma GPx3 significantly correlated with $(C)$ poor overall survival and (D) poor disease free survival of HCC patients' post-transplantation. The case number depended on the availability of clinical samples. 
Lower plasma GPx3 was identified as an independent predictor for poor overall survival of HCC patients after liver transplantation

The independent predictor for overall survival was further identified by Cox proportional hazard regression analysis (Table 1). In univariable analysis, lower plasma GPx3 at day 1 post-transplantation (HR=7.237, 95\%CI: 2.416-21.676, $P=0.000)$, lower plasma GPx3 at day 7 post-transplantation $(\mathrm{HR}=3.240$, 95\%CI: 1.152-9.115, $P=0.026)$, beyond UCSF criteria ( $\mathrm{HR}=2.585, \quad 95 \% \mathrm{CI}:$ 1.151-5.802, $P=0.021)$, higher number of tumor nodules $(\mathrm{HR}=2.660,95 \% \mathrm{CI}$ : $1.163-6.086, P=0.020)$ were all significantly associated with poor overall survival. Other conventional prognostic factors, such as higher AFP, beyond Milan criteria, larger tumor size and venous infiltration, were not significantly associated with overall survival (Table S2). Most importantly, in multivariable analysis, only plasma GPx3 at day 1 post-transplantation $(\mathrm{HR}=4.528,95 \% \mathrm{CI}$ : 1.026-19.988, $P=0.046)$ was identified as an independent predictor for overall survival of HCC patients (Table 1).

Table 1: Cox proportional hazard regression model for overall survival analysis.

\begin{tabular}{|c|c|c|c|c|}
\hline & \multicolumn{2}{|l|}{ Univariable analysis } & \multicolumn{2}{|c|}{ Multivariable analysis } \\
\hline & $\mathrm{HR}(95 \% \mathrm{CI})$ & $P$ & $\mathrm{HR}(95 \% \mathrm{CI})$ & $P$ \\
\hline \multicolumn{5}{|l|}{ Plasma GPx3 (Day 1) } \\
\hline Low vs High level & $7.237(2.416-21.676)$ & $0.000^{*}$ & $4.528(1.026-19.988)$ & $0.046^{*}$ \\
\hline \multicolumn{5}{|c|}{ Plasma GPx3 (Day 7) } \\
\hline Low vs High level & $3.240(1.152-9.115)$ & $0.026^{*}$ & $1.008(0.285-3.568)$ & 0.991 \\
\hline \multicolumn{5}{|l|}{ UCSF criteria } \\
\hline Beyond vs Within & $2.585(1.151-5.802)$ & $0.021^{*}$ & $0.520(0.069-3.943)$ & 0.527 \\
\hline \multicolumn{5}{|c|}{ Number of tumor nodules } \\
\hline$\geq 3$ vs $<3$ & $2.660(1.163-6.086)$ & $0.020^{*}$ & $3.048(0.422-22.023)$ & 0.269 \\
\hline
\end{tabular}

HR: Hazard ratio. CI: Confidence interval. ${ }^{*} P<0.05$.

\section{Over-expression of GPx3 significantly suppressed migration, invasiveness and metastasis of HCC cells}

In order to explore the functional role of GPx3 in liver cancer, HCC cell line (MHCC97L) with stable over-expression of GPx3 was established (Figure S2). Although over-expression of GPx3 had little effect on the migration of HCC cells in wound healing assay (Figure $3 \mathrm{~A}$ and S3), it significantly inhibited the invasiveness of HCC cells in Matrigel invasion assay (Figure 3B). It implied that GPx3 suppressed HCC invasion through suppressing degradation of extracellular matrix, but not through suppressing migration of HCC cells. Moreover, in order to explore the effect of GPx3 on HCC invasiveness, we established the orthotopic nude mice liver cancer model. Five weeks after the tumor implantation, the animals were sacrificed for samples collection and further analysis. We observed that the over-expression of GPx3 significantly suppressed intra-hepatic spread of tumor cells (Figure 3C, left panel). H\&E staining showed that more metastatic tumor nodules were observed in the control group compared with GPx3 over-expression group (Figure $3 \mathrm{C}$, right panel). Furthermore, the lung metastasis was significantly inhibited by over-expression of GPx3 in nude mice lung metastasis model (Figure 3D).

\section{Over-expression of GPx3 significantly suppressed HCC invasiveness in a living animal by real-time intravital imaging}

In order to investigate the suppressive role of GPx3 in HCC invasiveness in vivo, the ectopic nude mice liver cancer model with dorsal window chamber was established (Figure 4A). The real-time intravital images were captured through the dorsal window chamber under confocal microscope. This intravital imaging platform enabled us to kinetically observe the tumor behavior and directly see the tumor cells in a living animal. We found that over-expression of GPx3 significantly suppressed HCC invasiveness at different time points after tumor established (Figure 4B and S4A). HCC cells gradually invaded into the surrounding connective tissues in the control group. However, the boundary between HCC cells and surrounding connective tissues appeared intact in the GPx3 over-expression group. Furthermore, according to $3 \mathrm{D}$ reconstructed images, we could clearly observe that more tumor cells invaded into the surrounding connective tissues in Z-axis in the control group as early as 2 weeks after innoculation (Figure 4C, Movie S1 and Movie S3). In the contrast, the invasiveness of HCC cells with over-expression of GPx3 was significantly inhibited (Figure 4C and S4B, Movie S2 and Movie S4).

\section{GPx3 suppressed HCC invasiveness through inhibition of JNK-cJun-MMP2 signaling pathway in HCC cells, animal model and clinical samples}

Metalloproteinases (MMPs) are required to facilitate the degradation of extracellular matrix in the process of tumor invasion. Among the three commonly down-regulated subtypes of MMPs (MMP2, MMP7 and MMP9) in liver cancer, only MMP2 was most significantly suppressed upon GPx3 over-expression (Figure 5A). It has been reported that MMP2 expression is regulated by MAP Kinase signaling pathway. In our study, we found that the nuclear translocation of JNK (one of MAP Kinases) was significantly inhibited upon over-expression of GPx3 (Figure 5B). Furthermore, the activation of JNK and its down-stream target c-Jun were significantly inhibited upon over-expression of GPx3 (Figure 5C). It suggested that the tumor suppressive function of GPx3 may be mediated through JNK-cJun-MMP2 signaling pathway (Figure S5). 

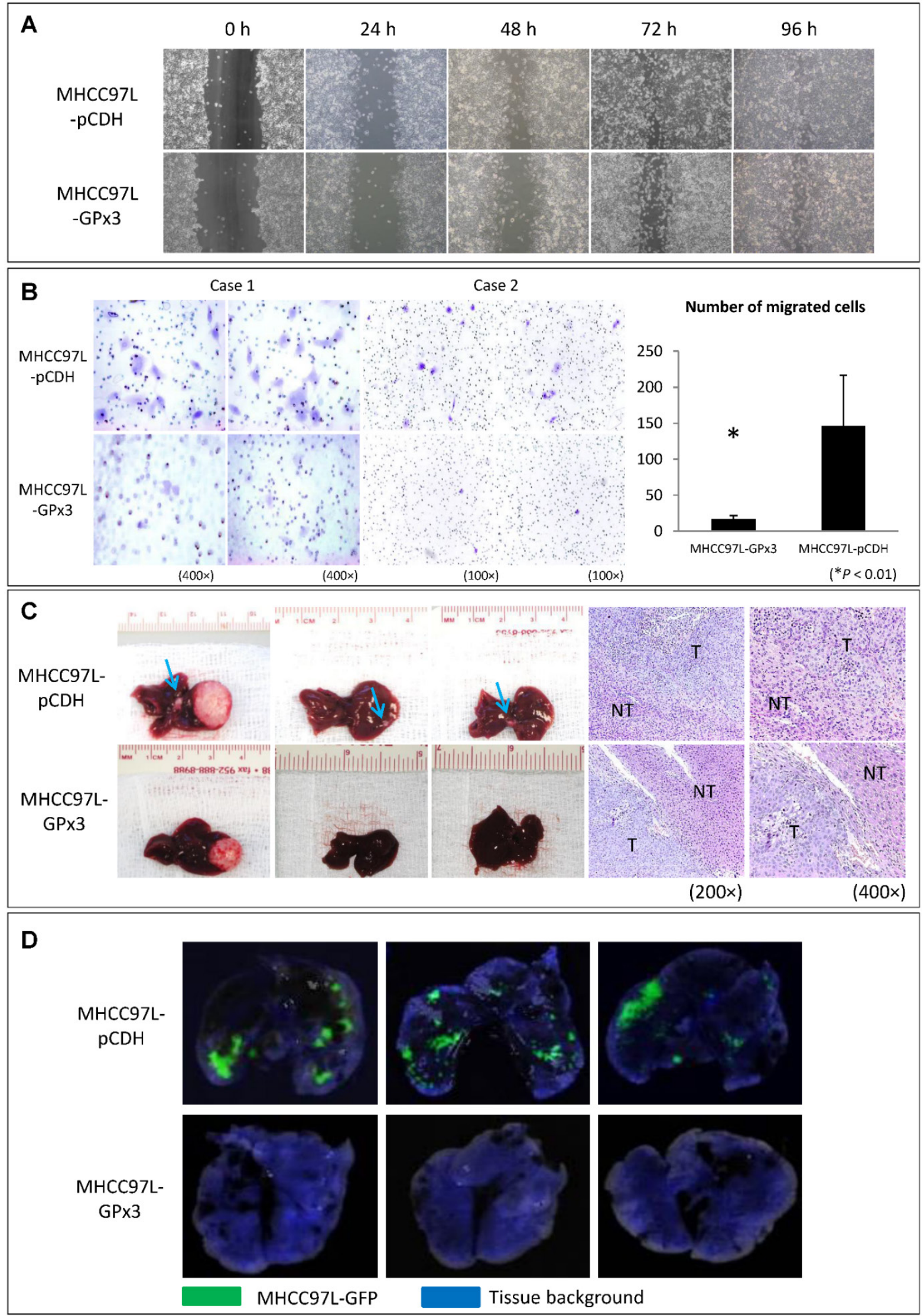

Figure 3. Over-expression of GPx3 significantly suppressed migration, invasiveness and metastasis of HCC cells. (A) Over-expression of GPx3 significantly suppressed migration of HCC cells in wound healing assay. (B) Over-expression of GPx3 significantly suppressed invasiveness of $\mathrm{HCC}$ cells in Matrigel invasion assay, $* P<0.01$. The error bar represented as mean \pm SD. (C) Over-expression of GPx3 significantly inhibited intra-hepatic tumor spread in orthotopic nude mice liver cancer model $(n=6)$. (D) Over-expression of GPx3 significantly inhibited lung metastasis of HCC cells injected through tail vein of nude mice $(n=3)$. 

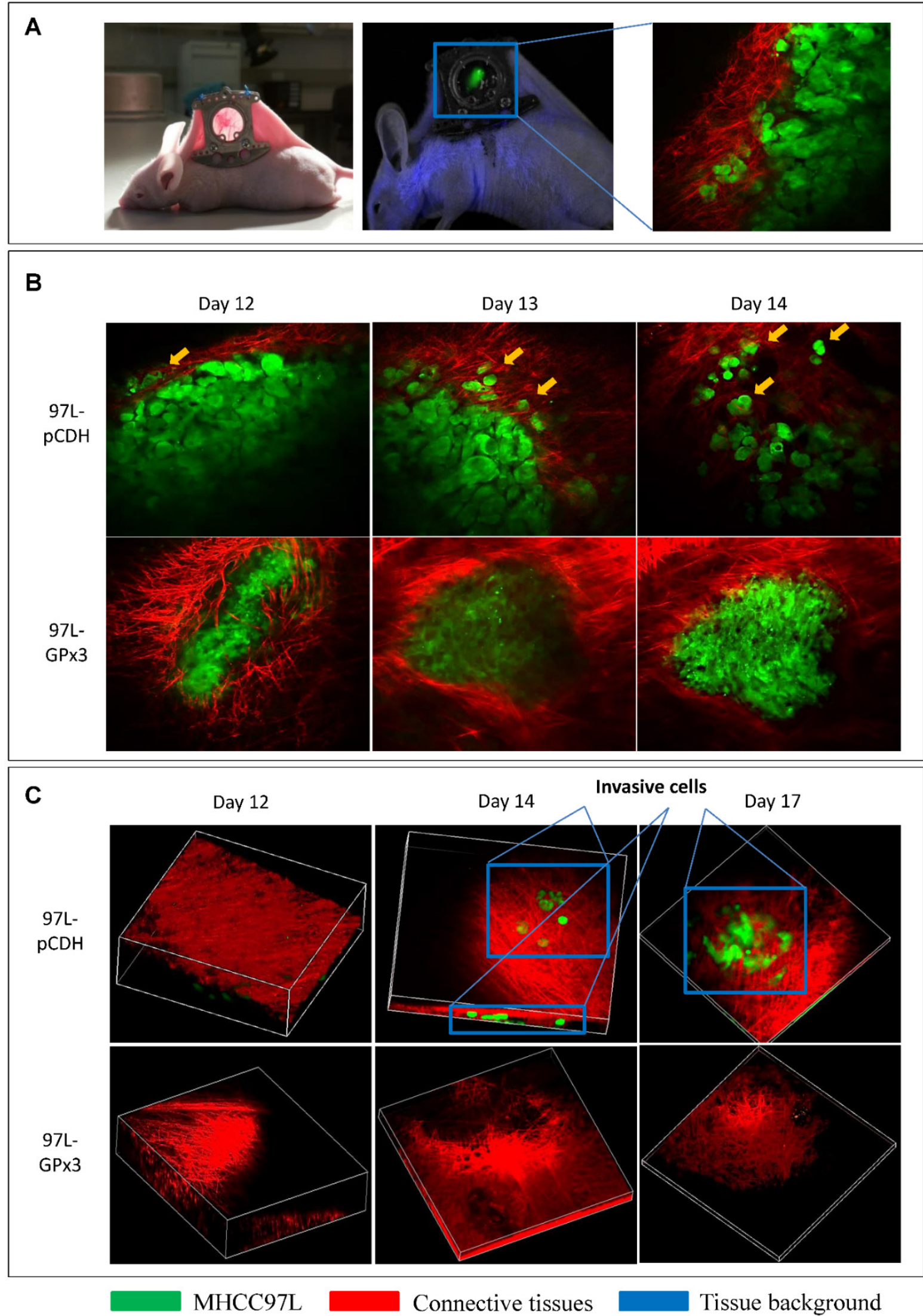

Figure 4. Real-time intravital imaging showed that over-expression of GPx3 significantly suppressed HCC invasiveness. (A) Ectopic nude mice liver cancer model with dorsal window chamber was established. (B) HCC cells gradually invaded into surrounding connective tissues at different time points in the control group. In the contrast, HCC cells with over-expression of GPx3 remained intact. Yellow arrow heads indicated invasive tumor cells. (C) Three-dimension reconstructed images showed that $\mathrm{HCC}$ cells invaded into surrounding connective tissues in Z-axis as early as 2 weeks after innoculation. However, the invasiveness of HCC cells with over-expression of GPx 3 was significantly inhibited. 

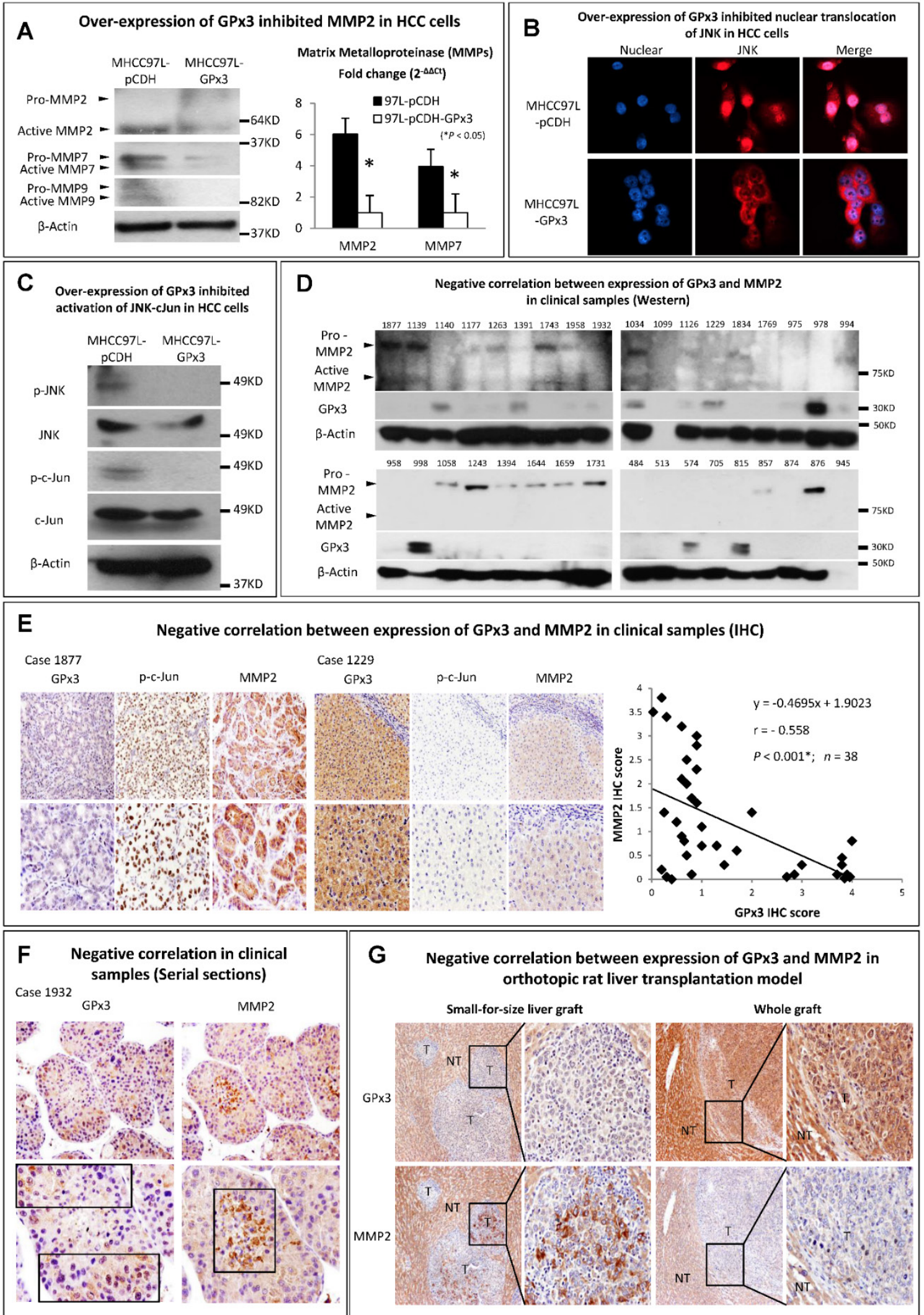

Figure 5. The suppressive function of GPx3 may be mediated through inhibition of JNK-cJun-MMP2 signaling pathway. (A) Expression of MMP2 was most significantly suppressed upon GPx3 over-expression in HCC cells in protein (left panel) and mRNA level (right panel). (B) Nuclear translocation of JNK was significantly attenuated upon GPx3 over-expression in HCC cells. The error bar represented as mean \pm SD. (C) Activation of JNK-cJun signaling pathway was significantly inhibited upon GPx3 over-expression in HCC cells. Clinically, MMP2 expression negatively correlated with GPx3 expression in tumor tissues collected from HCC patients during liver transplantation: (D) Western-blot (E) IHC staining. (F) The negative correlation between MMP2 and GPx3 expression was further validated in the clinical serial sections. (G) In orthotopic rat liver transplantation model with tumor development, the negative correlation between MMP2 and GPx3 expression was also confirmed. More intra-hepatic tumor spread was observed in small-for-size liver graft in which GPx3 was significantly down-regulated while MMP2 was up-regulated. The opposite trend could be observed in the whole graft. 
We further validated the results in the clinical samples of tumor tissues from 38 HCC patients who have undergone liver transplantation. We found that MMP2 expression negatively correlated with GPx3 expression (Figure 5D, 5E, S6 and S7). Moreover, the negative correlation between GPx3 and MMP2 expression in IHC staining was statistically significant $(r=-0.558, P<0.001$, Figure 5E, right panel) according to linear regression analysis. The negative correlation was also validated in distribution of GPx3 and MMP2 in serial sections. We found that GPx3 was significantly down-regulated while the MMP2 was significantly up-regulated in the same area of serial sections (Figure 5F).

In order to avoid the clinical confounding factors, we established a rat orthotopic liver transplantation model with tumor development. We found that intra-hepatic tumor metastasis was significantly higher in small-for-size liver graft compared with whole graft (Figure 5G). This finding is consistent with our previous study that small-for-size liver graft may be favorable for tumor growth [6, 21]. Moreover, GPx3 was significantly down-regulated while MMP2 was significantly up-regulated in small-for-size liver graft (Figure 5G, left panel). The opposite trend could be observed in the whole graft (Figure 5G, right panel). It implied that down-regulation of GPx3 and up-regulation of MMP2 may contribute to tumor progression in small-for-size liver graft.

\section{Discussion}

We identified that down-regulation of GPx3 in small-for-size liver graft was significantly associated with HCC invasiveness in a rat model and further found that lower plasma GPx3 was an independent predictor for poor overall survival of HCC patients after liver transplantation. Moreover, we found that GPx3 was not only a predictor for prognosis, but also suppressed HCC invasiveness through JNK-cJun-MMP2 signaling pathway. Therefore, in addition to prognostic value, GPx3 may possess the therapeutic potential targeting at $\mathrm{HCC}$ recurrence after liver transplantation.

Although higher AFP level [22, 23], larger tumor size [24, 25], venous infiltration [22, 23], advanced pTNM stage [26], beyond UCSF [27] or Milan [23] criteria are normally applied as prognostic markers for tumor recurrence after liver resection, these factors were not identified as independent predictors for overall survival after liver transplantation in our study. It might be attributed to the different mechanisms of tumor recurrence between liver transplantation and resection. The whole diseased liver was completely removed in transplantation. The only source for tumor recurrence is the tumor cells remained in circulation or other organs. Tumor recurrence highly depends on the environmental factors in the newly implanted liver. Therefore, the plasma GPx3, which is negatively associated with inflammatory environment in the liver graft, was identified to be correlated with prognosis post-transplantation. Recently, a series of novel markers for HCC recurrence after liver transplantation were identified, such as CD147 [28], HOTAIR [29], YAP [30] and MALAT1 [31]. However, they may not possess the therapeutic effect to prevent tumor recurrence. GPx3 may possess both prognostic and therapeutic value for HCC patients after liver transplantation.

The conventional animal study for investigation of tumor invasiveness always drew the conclusion based on the observation at end time point or sacrifice. However, the tumor invasiveness in a living animal is a dynamic process and could initiate at very early stage. Although the tumor cells labeled with bioluminescence could be kinetically detected, it cannot visualize intra-hepatic tumor invasion and illustrate the invasion process in a single cell level. Currently available optical techniques of functional imaging, such as MRI, PET and CT, are also limited by restricted penetration depth imposed by tissue turbidity, which avoids the possibility of utilizing high resolution microscope [13]. Real-time intravital imaging with dorsal window chamber enables us to kinetically observe the tumor behavior and directly see the tumor cells in a living animal. We could clearly observe that tumor cells gradually invaded into the surrounding connective tissue at different time points in a single cell level under confocal microscope. Furthermore, we could explore the process of tumor invasion in a 3-dimenssional view. Using this technique, we kinetically observed that over-expression of GPx3 significantly suppressed HCC invasiveness in a living animal.

Matrix metalloproteinase family (MMPs) is required for the tumor cells to degrade the extracellular matrix and to facilitate tumor invasiveness [32]. Thus, the MMPs inhibitors were applied in many clinical trials for cancer therapy [33]. However, these trials have been proven disappointing so far [34]. It is probably due to that MMPs are involved at early stage of tumor progression [34, 35]. In our study, the real-time intravital imaging also showed that the HCC cells invaded into surrounding tissue as early as two weeks after innoculation. That could partially explain the failure of application of MMPs inhibitor in clinical trials with end-stage tumor [35]. Our study showed that the lower GPx3 level during one-week post-transplantation significantly 
correlated with tumor recurrence and poor survival of HCC patients. Therefore, application of GPx3 administration at early phase post-operation may provide new therapeutic strategy for HCC patients after liver transplantation.

In conclusion, we identified that down-regulation of GPx3 was associated with HCC invasiveness in a rat liver transplantation model. Clinically, plasma GPx3 was significantly lower in the patients with tumor recurrence after liver transplantation and the lower plasma GPx3 was identified as an independent predictor for poor overall survival. Moreover, over-expression of GPx3 significantly suppressed migration, invasiveness and metastasis of HCC cells. Most importantly, the real-time intravital imaging has shown that over-expression of GPx3 kinetically suppressed HCC invasiveness in a living animal. Furthermore, the tumor suppressive function of GPx3 may be mediated through inhibition of JNK-cJun-MMP2 signaling pathway. Based on the advantage of the dual role of GPx3 in suppression of oxidative stress and liver cancer, the application of GPx3 administration may be a "one stone for two birds" strategy to attenuate hepatic IR injury and prevent tumor recurrence after liver transplantation. The suppressive role of GPx3 in inflammation in liver graft injury is worthwhile for further investigation.

\section{Supplementary Material}

Additional File 1:

Supplementary tables and figures.

http://www.thno.org/v06p1934s1.pdf

Additional File 2:

Movie S1. http:/ / www.thno.org/v06p1934s2.avi

Additional File 3:

Movie S2. http:/ / www.thno.org/v06p1934s3.avi

Additional File 4:

Movie S3. http:/ / www.thno.org/v06p1934s4.avi

Additional File 5:

Movie S4. http:/ / www.thno.org/v06p1934s5.avi

\section{Abbreviations}

HCC, hepatocellular carcinoma; GPx3, glutathione peroxidase 3; MHCC97L, New human hepatocellular carcinoma cell line with metastatic potential; MMP, Metalloproteinases; JNK, c-Jun N-terminal kinases.

\section{Acknowledgement}

This study was supported by the Collaborative Research Fund (HKU3/CRF/11R \& C7027-14GF) and General Research Funding (75011M, 17115515\& 17115614) of the Research Grant Council, Hong Kong; A National Science Foundation of China (NSFC) grant
(No.81470903 \& 81572945); Small project funding for Basic Research (201409176092), HKU.

\section{Competing Interests}

The authors have declared that no competing interest exists.

\section{References}

1. Lo CM, Fan ST, Liu CL, et al. Living donor versus deceased donor liver transplantation for early irresectable hepatocellular carcinoma. Br J Surg. 2007; 94: 78-86.

2. Fisher RA, Kulik LM, Freise CE, et al. Hepatocellular carcinoma recurrence and death following living and deceased donor liver transplantation. Am J Transplant. 2007; 7: 1601-8.

3. Kulik LM, Fisher RA, Rodrigo DR, et al. Outcomes of living and deceased donor liver transplant recipients with hepatocellular carcinoma: results of the A2ALL cohort. Am J Transplant. 2012; 12: 2997-3007.

4. Ling CC, Ng KT, Shao Y, et al. Post-transplant endothelial progenitor cell mobilization via CXCL10/CXCR3 signaling promotes liver tumor growth. J Hepatol. 2014; 60: 103-9.

5. Man K, Lo CM, Xiao JW, et al. The significance of acute phase small-for-size graft injury on tumor growth and invasiveness after liver transplantation. Ann Surg. 2008; 247: 1049-57.

6. Man $\mathrm{K}$, Shih $\mathrm{KC}, \mathrm{Ng} \mathrm{KT}$, et al. Molecular signature linked to acute phase injury and tumor invasiveness in small-for-size liver grafts. Ann Surg. 2010; 251: 1154-61.

7. Qi X, Ng KT, Lian QZ, et al. Clinical significance and therapeutic value of glutathione peroxidase 3 (GPx3) in hepatocellular carcinoma. Oncotarget. 2014; 5: 11103-20.

8. Ottaviano FG, Tang SS, Handy DE, et al. Regulation of the extracellular antioxidant selenoprotein plasma glutathione peroxidase (GPx-3) in mammalian cells. Mol Cell Biochem. 2009; 327: 111-26.

9. Yu YP, Yu G, Tseng G, et al. Glutathione peroxidase 3, deleted or methylated in prostate cancer, suppresses prostate cancer growth and metastasis. Cancer Res. 2007; 67: 8043-50.

10. Zhang X, Yang JJ, Kim YS, et al. An 8-gene signature, including methylated and down-regulated glutathione peroxidase 3, of gastric cancer. Int J Oncol. 2010; 36: 405-14.

11. Peng DF, Razvi M, Chen $\mathrm{H}$, et al. DNA hypermethylation regulates the expression of members of the Mu-class glutathione S-transferases and glutathione peroxidases in Barrett's adenocarcinoma. Gut. 2009; 58: 5-15.

12. Sreekanthreddy P, Srinivasan H, Kumar DM, et al. Identification of potential serum biomarkers of glioblastoma: serum osteopontin levels correlate with poor prognosis. Cancer Epidemiol Biomarkers Prev. 2010; 19: 1409-22.

13. Palmer GM, Fontanella AN, Shan S, et al. In vivo optical molecular imaging and analysis in mice using dorsal window chamber models applied to hypoxia, vasculature and fluorescent reporters. Nat Protoc. 2011; 6: 1355-66.

14. Laschke MW, Vollmar B, Menger MD. The dorsal skinfold chamber: window into the dynamic interaction of biomaterials with their surrounding host tissue. Eur Cell Mater. 2011; 22: 147-64; discussion 64-7.

15. Ng KT, Lo CM, Guo DY, et al. Identification of transmembrane protein 98 as a novel chemoresistance-conferring gene in hepatocellular carcinoma. Mol Cancer Ther. 2014; 13: 1285-97.

16. Geng $\mathrm{W}$, Lo $\mathrm{CM}, \mathrm{Ng} \mathrm{KT}$, et al. Interferon-gamma inducible protein 10 (IP10) induced cisplatin resistance of HCC after liver transplantation through ER stress signaling pathway. Oncotarget. 2015; 6: 28042-56.

17. Li CX, Wong BL, Ling CC, et al. A novel oxygen carrier "YQ23" suppresses the liver tumor metastasis by decreasing circulating endothelial progenitor cells and regulatory T cells. BMC Cancer. 2014; 14: 293.

18. Yeung OW, Lo CM, Ling CC, et al. Alternatively activated (M2) macrophages promote tumour growth and invasiveness in hepatocellular carcinoma. J Hepatol. 2015; 62: 607-16.

19. $\mathrm{Ng} \mathrm{KT}$, Guo DY, Cheng Q, et al. A garlic derivative, S-allylcysteine (SAC), suppresses proliferation and metastasis of hepatocellular carcinoma. PLoS One. 2012; 7: e31655.

20. Man K, Ng KT, Xu A, et al. Suppression of liver tumor growth and metastasis by adiponectin in nude mice through inhibition of tumor angiogenesis and downregulation of Rho kinase/IFN-inducible protein 10/matrix metalloproteinase 9 signaling. Clin Cancer Res. 2010; 16: 967-77.

21. Zimmerman MA, Ghobrial RM, Tong MJ, et al. Recurrence of hepatocellular carcinoma following liver transplantation: a review of preoperative and postoperative prognostic indicators. Arch Surg. 2008; 143: 182-8; discussion 8.

22. Vivarelli M, Cucchetti A, Piscaglia F, et al. Analysis of risk factors for tumor recurrence after liver transplantation for hepatocellular carcinoma: key role of immunosuppression. Liver Transpl. 2005; 11: 497-503.

23. Cescon M, Ravaioli M, Grazi GL, et al. Prognostic factors for tumor recurrence after a 12-year, single-center experience of liver transplantations in patients with hepatocellular carcinoma. J Transplant. 2010; 2010. 
24. Ataide EC, Boin IF, Almeida JR, et al. Prognostic factors for hepatocellular carcinoma recurrence: experience with 83 liver transplantation patients. Transplant Proc. 2011; 43: 1362-4.

25. Kim YS, Lim HK, Rhim H, et al. Recurrence of hepatocellular carcinoma after liver transplantation: patterns and prognostic factors based on clinical and radiologic features. AJR Am J Roentgenol. 2007; 189: 352-8.

26. Lai Q, Avolio AW, Lerut J, et al. Recurrence of hepatocellular cancer after liver transplantation: the role of primary resection and salvage transplantation in East and West. J Hepatol. 2012; 57: 974-9.

27. Parfitt JR, Marotta P, Alghamdi M, et al. Recurrent hepatocellular carcinoma after transplantation: use of a pathological score on explanted livers to predict recurrence. Liver Transpl. 2007; 13: 543-51.

28. Zhang Q, Chen X, Zhou J, et al. CD147, MMP-2, MMP-9 and MVD-CD34 are significant predictors of recurrence after liver transplantation in hepatocellular carcinoma patients. Cancer Biol Ther. 2006; 5: 808-14.

29. Yang Z, Zhou L, Wu LM, et al. Overexpression of long non-coding RNA HOTAIR predicts tumor recurrence in hepatocellular carcinoma patients following liver transplantation. Ann Surg Oncol. 2011; 18: 1243-50.

30. Li H, Wang S, Wang G, et al. Yes-associated protein expression is a predictive marker for recurrence of hepatocellular carcinoma after liver transplantation. Dig Surg. 2014; 31: 468-78.

31. Lai MC, Yang Z, Zhou L, et al. Long non-coding RNA MALAT-1 overexpression predicts tumor recurrence of hepatocellular carcinoma after liver transplantation. Med Oncol. 2012; 29: 1810-6.

32. Brinckerhoff CE, Matrisian LM. Matrix metalloproteinases: a tail of a frog that became a prince. Nat Rev Mol Cell Biol. 2002; 3: 207-14.

33. Bramhall SR, Hallissey MT, Whiting J, et al. Marimastat as maintenance therapy for patients with advanced gastric cancer: a randomised trial. $\mathrm{Br} \mathrm{J}$ Cancer. 2002; 86: 1864-70.

34. Coussens LM, Fingleton B, Matrisian LM. Matrix metalloproteinase inhibitors and cancer: trials and tribulations. Science. 2002; 295: 2387-92.

35. Egeblad M, Werb Z. New functions for the matrix metalloproteinases in cancer progression. Nat Rev Cancer. 2002; 2: 161-74. 